\title{
1x1 Trainer with Handwriting Recognition
}

\author{
https://doi.org/10.3991/ijim.v12i2.7714 \\ M. Rabko, and M. Ebner $\left.{ }^{\bowtie}\right)$ \\ Graz University of Technology, Graz, Austria \\ mebner agmx a at
}

\begin{abstract}
Nowadays, computers and mobile devices play a huge role in our daily routines; they are used at work, for private purposes and even at school. Moreover, they are used as support for different kinds of activities and task, like for example, learning applications. The interaction of these applications with a computer is based on predefined input methods, whereas a touchscreen facilitates direct input via handwriting by using a finger or a pen.

This paper deals with the invention of a mobile learning application, which is supposed to facilitate children's learning of simple multiplication. The aim of this paper is to collect the data of children' experiences using interactive handwriting on mobile devices. In order to gain this data, a school class of the school "Graz-Hirten" was tested and afterwards for evaluation purposes interviewed. The results of these usability tests have shown that children perceived handwriting via finger on screen as quite positive.
\end{abstract}

Keywords-handwriting recognition, convolutional neural network, multiplication table, learning, children, mobile learning

\section{Introduction}

Handwriting is an individual skill and used for communication such as sharing emotions and opinions. Everybody has their own individual way and style of writing letters, numbers and signs. Despite the fact that every person writes quite individually, people recognize and understand what is written. Keypads make it possible to enter letters, numbers and signs into electronic devices. Because of predefined and preprogrammed input methods, these devices are able to process written language. However, input via handwriting makes this process quite more difficult since devices cannot process or interpret it.

Thus handwriting recognition is a subarea of pattern recognition. The idea is to "teach" a device how to process and recognize handwriting. Devices are supposed to notice changes in their environment and to recognize patterns and subsequently to make decisions [1].

In the recognition of handwriting it can be differentiated between "online" and "offline" recognition [2]. On the one hand, in relation to online recognition, handwriting is being processed during the input. The information that is collected during this pro- 
cess is of great importance. On the other hand, in relation to offline recognition, the information is only processed and recognized at a later point.

Handwriting recognition is used in many different areas such as Personal Digital Assistants (PDA), banking, post services [3] and even learning applications for children.

The aim of this paper is to show how young schoolchildren react to this input method and to evaluate test results of usability tests of handwriting recognition. Moreover, it will be examined if this input method is favoured by children when it is compared to the conventional keypad input method. In order to get these results, a learning application with included offline handwriting detection has been created and evaluated. The invented application should facilitate children learning of simple multiplication and is based on the already existing application " $1 \mathrm{x} 1$ trainer" which was created by Graz University of Technology. The app itself will be another LearningAnalytics-Application [14] because each calculation will also be sent to a central database and analysed for further trainings.

\section{$2 \quad$ Prototyping}

From a research perspective the study is following the method of prototyping with proof of concept afterwards. With other words an application was built which fulfils the defined goals and was given to children for a first field test. After a thoroughly study of the app store only few math apps were found with the possibility of handwriting numbers, namely: ABCFunkid Calculus Lite, Write Math, Handwriting Math Training, Handwriting Math Training for Kids and Todo Math. All apps in common were that the handwriting works not perfect and so an improvement seems to be obviously.

The already mentioned „1x 1 trainer" is a mathematical learning application which facilitates the learning and studying of simple multiplications [4]. The special feature of the new developed app is that it is able to recognize and process handwritten numbers. Answers to the calculation tasks can be written onto the touchscreen of a mobile device. The application is created for a target group of children between 6 and 12 years. One main idea was that children do not get distracted or confused by too much text [5]. Children who use this app should be attracted and motivated by the design of the user interface. Therefore, the main focus of this application was the clear structured user interface and the abandonment of visual elements.

The application has two playing modes, which share the same principle. The only difference between these two modes is the variety of tasks and the option of saving the learning progress. In the first mode, a server, which runs in the background, chooses the tasks with the help of an algorith. In contrast, in the other mode tasks are chosen randomly.

There are 20 calculation tasks, which the player is supposed to answer within 60 seconds. The faster the task is solved, the more points can be scored. At the end of the game, the solved tasks and the gained points are summed up which is supposed to support the learning effect. 


\section{Application}

The newly invented learning game uses for the recognition of handwritten numbers a Convolutional Neural Network consisting of multiple layers since this kind of neural network scores the best results concerning the recognition of handwritten numbers [7][8]. The so-called CNN consists out of two Convolution-layers, to which the MaxPooling-layer is connected, and in addition, a fully connected layer. The Figure 1 shows the structure of the used CNN.

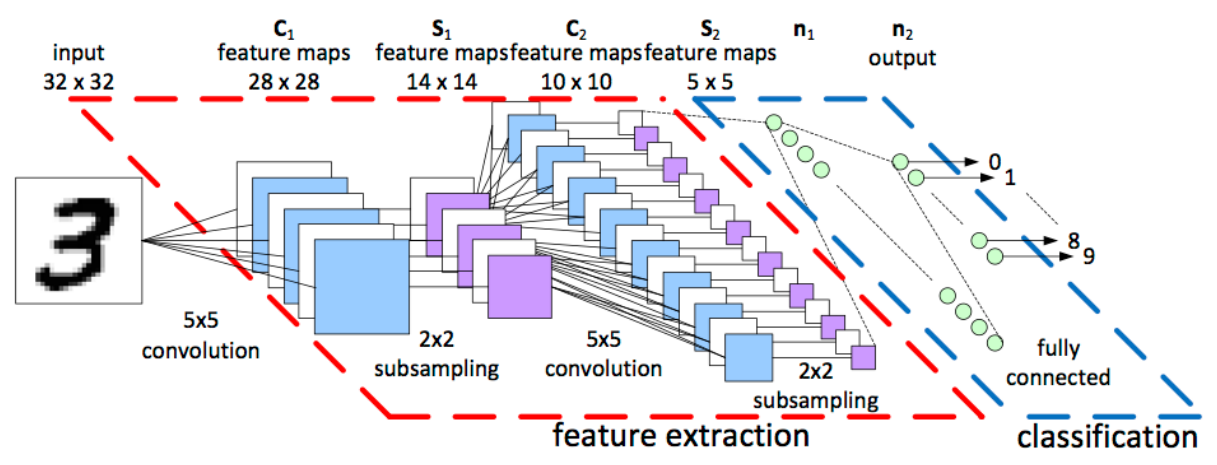

Fig. 1. Convolutional neural network

\subsection{Training}

The defined CNN has to be trained with suitable and sufficient data in order to recognise handwritten numbers. TensorFlow and the MNIST database are used for this process of training the neural network. TensorFlow is a machine-learning library developed by Google [9]. This library builds the neural network and provides it with input of the MNIST database. The MNIST database consists of images of handwritten numbers of the size of $28 \times 28$ pixel. It includes 60.000 images for the training and further 10.000 images to test the new-configured neural network [10].

The duration of the whole process described here depends on the processing power of the device and takes approximately 30 minutes. The CNN scores a recognition rate of $99 \%$ over all test images.

\subsection{Adaptation}

The TensorFlow script saves weights and bias of all layers of the trained convolutional neural network in separate files. Therefore, in order to apply the CNN with the saved files, the " $1 \mathrm{x} 1$ trainer" uses the "Basic neural network subroutines" (BNNS). Apple introduced the BNNS with the iOS 10. It is part of the Accelerate framework and helps the developer to run neural networks in iOS-applications. However, BNNS supports only neural networks, which are already trained with test data. 
The import of the data written with TensorFlow carries the risk that the neural network does not function properly. There are differences between TensorFlow and BNNS concerning the arrangement of pixel values within an array. BNNS expects pixel values to be arranged in row by row, by channels within a file. TensorFlow arranges data in a completely different way - images are arranged in pixel per channel by pixel per channel. In Figure 2 the differences in the arrangement of image data are shown. Applied matrix operations within the training script solve these problems.

The writing of numbers is allowed in two predefined boxes whereas each box represents a number. The advantage of this is that the application does not have to filter single numbers from the input.

Moreover, the application is, due to this, clearly arranged and easy to operate. The Figure 3 shows these two boxes for the input of handwritten numbers.

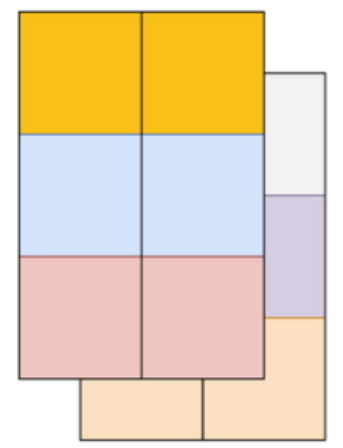

(a) Array of pixel data

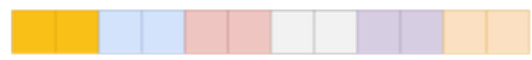

(b) BNNS - Array

\section{(c) Tensorflow - Array}

Fig. 2. Difference of the data representation between BNNS and Tensorflow.

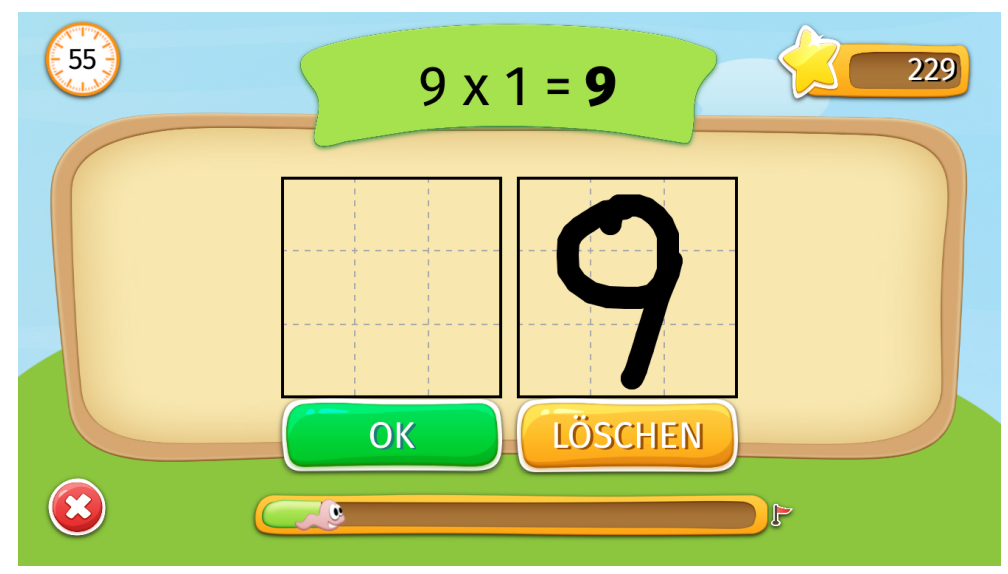

Fig. 3. $1 \mathrm{x} 1$ Trainer - Trainer mode 
After the user has written/drawn into one of the boxes, an UIImage is being processed. In the following step, the UIImage gets preprocessed for the recognition. This preprocessing includes the centering of the written number in the image and subsequently the scaling of the UIImage. This scaling is necessary because the convolutional neural network accepts only images sized $28 \times 28$ pixel.

After the preprocessing, the recognition starts with the UIImage. In the next step, the image runs through the following steps:

1. The first layer is a convolutional layer and calculates 32 features for each of the $5 \times 5$ kernel.

2. The second layer is a max-pooling layer with a $2 \times 2$ filter. This layer scales the image to $14 \times 14$ pixel.

3 . In the next step, another convolutional-layer calculates 64 features for each of the $5 \times 5$ kernel.

4. Afterwards, another max-pooling layer with a $2 \times 2$ filter scales the image to $7 \times 7$ pixel.

5. After these four layers, a fully connected layer can be found. This layer helps with the processing of the whole image.

6. In the last step, a softmax layer produces out of the resulting vector of the previous layer a vector with 10 entries. These ten entries represent numbers from zero to nine.

\subsection{General architecture of the app}

The application itself consist of 6 main screens, where users can navigate by pressing a back or forward button

1. Start screen: The user is welcomed and can choose between the trainer mode (logged-in mode) or just the training mode

2. Login screen: If the user chose the trainer mode he/she can provide his/her credentials. Afterwards each singe calculations will be sent to the server and saved for further learning analytics operations [13]

3. Onboarding screen: This screen is just for testing handwriting for the very first time. With other words a kind of help screen to show how to write the numbers of the result on the right place.

4. Main screen: The main screen (see Fig. 3) consist of the provided calculation and two boxes, where the result should be written by hand. Furthermore a small clock is presented as well as the current level points. Two buttons allow the use to confirm his/her result or to delete it (for example if the numbers were wrongly interpreted by the algorithm)

5. Summary screen: This screen summarizes the result of the trial -how many calculations done and how many of them correct. Finally the given points are displayed

6. High-score screen: In the end a high score list is presented 


\section{$4 \quad$ Evaluation}

The evaluation was conducted with an iPad-class of the elementary school GrazHirten following consequently a multi-level evaluation using the cut-off technique [11] [12]:

- First of all children are monitored by a research assistant during using the application in the classroom

- Second, interviews with the children were carried out in groups of 3-4 using the cut-off technique, due to children often are shy and did not express their real feelings about the application. Therefore a couple of statements were provided (see Table 1) and the pupils had to rank those statements using different symbols (smileys) providing their opinions against each other. So the research assistant did not directly ask his questions, but take the discussion amongst them as basis for his evaluation of the app.

For the final evaluation unfortunately only seven iPads could be used for testing the application since the other four iPads did not have the required iOS-version 10 installed. On the day where the evaluation took place, 20 of 25 pupils were attendant. The pupils were divided into three groups of 6-7. Each group played for 15 minutes with the " $1 \mathrm{x} 1$ trainer". However, the game had not been explained to the pupils beforehand. The reason for this was that the pupils should not be influenced by anything and come to their own opinion on this game since the latter is crucial for the opinion survey.

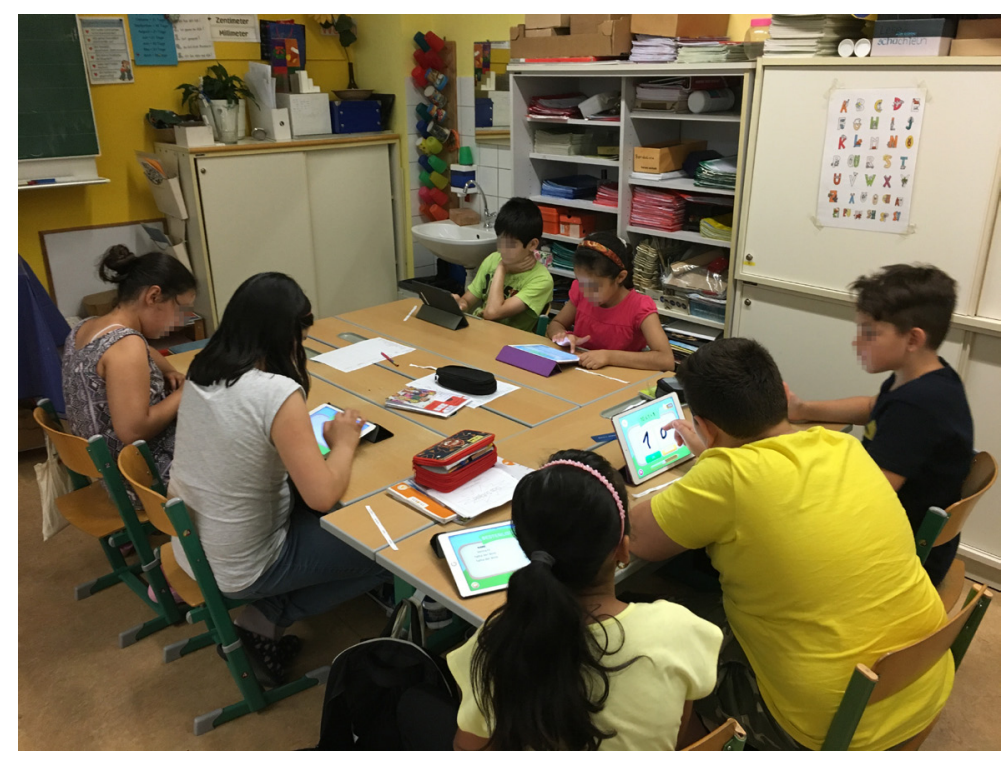

Fig. 4. Evaluation setup 
After each group had the chance to play with the " $1 \mathrm{x} 1$ trainer", the pupils were divided up again into smaller groups of 3-4. At the end, six groups of pupils were asked to provide feedback on the game. This feedback makes also the main part of the evaluation of the learning application. The pupils were asked to pick one of five smileys (as it can be seen in the Table 1) in order to assess a statement to be true for themselves (laughing smiley) or not true for themselves (sad smiley). The statements were carefully selected based on our experiences in similar research studies [11] [12].

These following statements had to be assessed by the pupils:

- I liked the game.

- I was able to get along with the game.

- Drawing numbers with my finger is fun for me.

- I would like to play the $1 \times 1$ trainer at home as well.

Table 1. Rating smileys

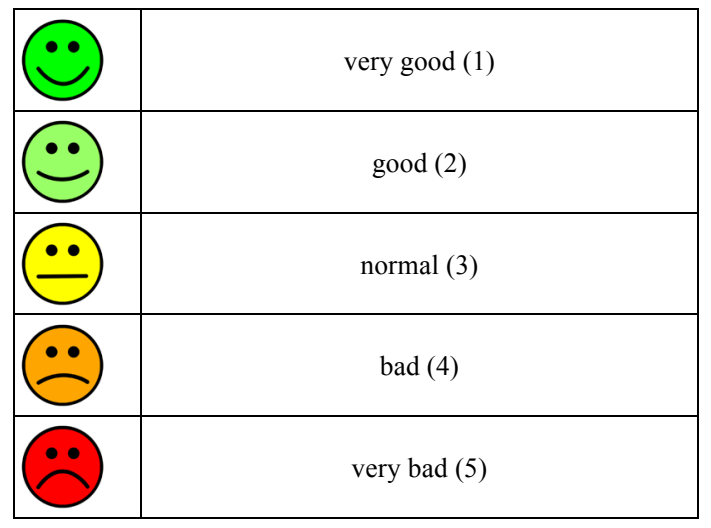

Table 2 gives an overview about the answer of the six groups concerning the given statements. The result of each group is pointed out as well as the average over all children.

Table 2. Evaluation results for each group

\begin{tabular}{|l|c|c|c|c|c|c|c|}
\hline \multicolumn{1}{|c|}{ Statement } & Group 1 & Group 2 & Group 3 & Group 4 & Group 5 & Group 6 & Average \\
\hline I liked the game & 1 & 1 & 2 & 1 & 1 & 1 & 1,17 \\
\hline $\begin{array}{l}\text { I was able to get along with } \\
\text { the game }\end{array}$ & 2 & 3 & 1 & 2 & 1 & 2 & 1,83 \\
\hline $\begin{array}{l}\text { Drawing numbers with my } \\
\text { finger is fun for me }\end{array}$ & 1 & 1 & 1 & 3 & 2 & 1 & 1,5 \\
\hline $\begin{array}{l}\text { I would like to play the 1x1 } \\
\text { trainer at home as well }\end{array}$ & 1 & 1 & 3 & 4 & 1 & 1 & 1,83 \\
\hline
\end{tabular}

The research assistant also collected some statements of the children (Fig. 5), like "the game was fun" and "very interesting to play during a math lesson". Concerning the handwriting he recognized that "it is fun to write numbers by hand" but also "that 
it become stressful if the number is not written exactly". Some children can imagine "to play the game also at home", but "maybe it can become boring too". Furthermore children summarized that the "app is great for an iPad, but handwriting on iPhone is cumbersome". Overall it can be stated that they liked th app, also the idea of handwriting and just mentioned some concerns about the small display of an iPhone and few problems not-recognized numbers.

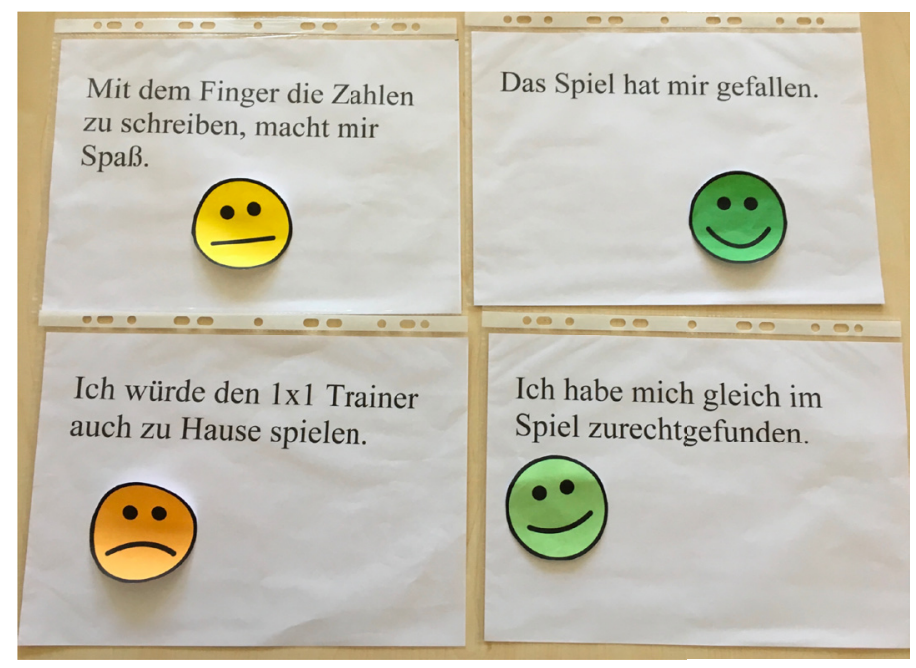

Fig. 5. Evaluation result of one group

\section{Discussion}

All in all, the evaluation of the prototype could be conducted without any greater problems. The " $1 \mathrm{x} 1$ trainer" as well as the connection to the server was functioning properly. The pupils had a lot of fun drawing numbers with their fingers. Even though the learning application got mainly positive feedback, the evaluation has also shown that the usability of the application needs improvement since several problems occurred, such as:

- Recognition of handwriting. Sometimes, written numbers were recognized wrongly which led to some confusion and frustration of the pupils. Especially the numbers 1 and 7 were confused by the application.

- Login in trainer mode. The login was a problem for some of the children since they tried to $\log$ in with their true names even though they had got the user data shortly before the test.

- Playing the game._Some pupils were overstrained when they started the game and did not know what to do with it.

- Single-digits._Another problem, which confused the children, was the question in which of the two boxes one-digit solutions should be written. However, the „1x1 trainer" accepts both boxes. 


\section{Conclusion}

The outcome of the evaluation shows clearly the potential of applications with handwriting recognition; especially of learning applications, which dispose this function. The pupils had a lot of fun drawing the numbers onto the screen. However, their opinions on whether they preferred handwriting or writing on a keypad were diverse. A reason why some of the pupils did not like handwriting could be the problems they had with the handwriting recognition since wrongly read numbers had a vast influence on the pupils' motivation.

Moreover, a positive side effect of using handwriting recognition in this application was that children put in more effort into drawing the numbers readable. This side effect was found due to the feedback survey and the assessment given by pupils.

In conclusion, the research for this paper supports that handwriting recognition should be used in learning applications for children, because it seems to motivate them. However, the success of an application strongly depends on the recognition rate as well as on the applied methods.

Of course the limitation of this study is that we just examined a prototype and its usability issues. The study did not examine if handwriting of numbers leads to a deeper learning or not. So we concentrate just on making an application more fun and easy to use. In further studies it must be investigated if those apps and learning analytics analyses help to learn the multiplication table more efficiently as first attempts have already shown [15].

Because of the mentioned problems with the " $1 \mathrm{x} 1$ trainer", some features of the application should be improved. The following approaches provide some ideas for improvements and extensions:

- Pretest. The "1x1 Trainer" does not use a pretest on the trainer mode yet to evaluate the existing knowledge of the user. The result of the lack of a pretest is that every user has to start with the simplest tasks. A pretest would motivate older pupils and those who are already quite good at simple multiplication.

- Handwriting Recognition._Although, the handwriting recognition is working with the application, there is still improvement possible. It might help to configure the applied neural network better or to extend it. Another possibility to improve the recognition would be to apply additional pre-processing techniques.

- Explanation of the Game. The playing modes are only explained in the main menu while the exact distribution is never mentioned at any point.

- Gamification. In order to maintain the motivation of the user, it would help to use gamification elements. Numbers that were read wrongly would not have such a huge impact on the players' motivation. 


\section{$7 \quad$ References}

[1] A. K. Jain, R. P. W. Duin, and J. Mao, „Statistical pattern recognition: A review,“ IEEE Transactions on pattern analysis and machine intelligence, vol. 22, no. 1, pp. 4-37, 2000. https://doi.org/10.1109/34.824819

[2] R. Plamondon and S. N. Srihari, „Online and off-line handwriting recognition: A comprehensive survey, "IEEE Transactions on pattern analysis and machine intelligence, vol. 22, no. 1, pp. 63-84, 2000. https://doi.org/10.1109/34.824821

[3] A. Priya, S. Mishra, S. Raj, S. Mandal, and S. Datta, „Online and offline character recognition: A survey,“ in Communication and Signal Processing (ICCSP), 2016 International Conference on, IEEE, 2016, pp. 0967-0970.

[4] Schön, M., Ebner, M., Kothmeier, G. (2012) It's Just About Learning the Multiplication Table, In Proceedings of the 2nd International Conference on Learning Analytics and Knowledge (LAK '12), Simon Buckingham Shum, Dragan Gasevic, and Rebecca Ferguson (Eds.). ACM, New York, NY, USA, 73-81. https://doi.org/10.1145/2330601.2330624

[5] Huber, S., \& Ebner, M. (2013). iPad Human Interface Guidelines for M-Learning. In Z.L. Berge and L.Y. Muilenburg (Eds.), Handbook of mobile learning. (pp. 318-328). New York: Routledge

[6] Ebner, M. (2015) Mobile Learning and Mathematics. Foundations, Design, and Case Studies. Crompton, H., Traxler, J. (ed.). Routledge. New York and London. pp. 20-32

[7] P. Y. Simard, D. Steinkraus, J. C. Platt, et al., „Best practices for convolutional neural networks applied to visual document analysis.," in ICDAR, vol. 3, 2003, pp. 958-962.

[8] D. Ciregan, U. Meier, and J. Schmidhuber, ,Multi-column deep neural networks for image classification, “ in Computer Vision and Pattern Recognition (CVPR), 2012 IEEE Conference on, IEEE, 2012, pp. 3642-3649.

[9] M. Abadi, A. Agarwal, P. Barham, E. Brevdo, Z. Chen, C. Citro, G. S. Corrado, A. Davis, J. Dean, M. Devin, et al., „Tensorflow: Large-scale machine learning on heterogeneous distributed systems," arXiv preprint arXiv:1603.04467, 2016.

[10] Y. LeCun, L. Bottou, Y. Bengio, and P. Haffner, „Gradient-based learning applied to document recognition,“ Proceedings of the IEEE, vol. 86, no. 11, pp. 2278-2324, 1998. https://doi.org/10.1109/5.726791

[11] Ebner, M., Schönhart, J. \& Schön, S. (2014). Experiences with iPads in primary schools. In: Profesorado, V 18, No 3, 161-173 (sept.-diciembre 2014).

[12] Lexow, S., Ebner, M. (2014) Development of a Collaborative Learning Game Using External Plastic Cards as an Input Device on an iPad, International Journal of Interactive Mobile Technologies (iJIM), Vol. 8, Nr. 2, p. 12-17

[13] Kraja, E., Taraghi, B. \& Ebner, M. (2017). The Multiplication Table as an innovative Learning Analytics Application. In J. Johnston (Ed.), Proceedings of EdMedia: World Conference on Educational Media and Technology 2017 (pp. 810-820). Association for the Advancement of Computing in Education (AACE).

[14] Ebner, M. (2015) Mobile Learning and Mathematics. Foundations, Design, and Case Studies. Crompton, H., Traxler, J. (ed.). Routledge. New York and London. pp. 20-32

[15] Ebner, M., Schön, M., Neuhold, B. (2014). Learning Analytics in basic math education first results from the field, eLearning Papers, 36, pp. 24-27 


\section{Authors}

M. Rabko studied Software Engineering and Management at Graz University of Technology (e-mail: michael.rabko@student.tugraz.at).

M. Ebner is the head of Department Educational Technology at Graz University of Technology and therefore responsible for all university wide e-learning activities. He holds an Adjunct Prof. on media informatics (research area: educational technology) and works also at the Institute of Interactive Systems and Data Science as senior researcher. His research focuses strongly on seamless learning, learning analytics, open educational resources, making and computer science for children. In 2012 he was awarded the New German Book Prize. 2015 he received the Austrian State Prize for Adult Education for his online course "Gratis Online Lernen" on the "iMoox" elearning platform. For publications as well as further research activities, please visit his website: http://martinebner.at

Article submitted 18 September 2017. Resubmitted 25 December 2017 and 10 January 2018. Final acceptance 06 March 2018. Final version published as submitted by the authors. 